\title{
Obesità, sindrome metabolica ipogonadismo maschile e rischio cardiovascolare
}

\section{Obesity, metabolic syndrome, male hypogonadism and cardiovascular risk}

\section{Giovanni Corona ${ }^{a, b, *}$, Francesco Lotti ${ }^{a}$, Alessandra Sforza ${ }^{b}$, Mario Maggi $^{a}$, Valerio Chiarini ${ }^{\text {b }}$}

\author{
a Unità di Andrologia, Dipartimento di Fisiopatologia Clinica, Università degli Studi di Firenze \\ ' UO Endocrinologia, Ospedale "Maggiore-Bellaria", Bologna
}

Ricevuto il 4 maggio 2009; accettato il 18 maggio 2009

disponibile online il 12 ottobre 2009

\section{KEYWORDS \\ Hypogonadism; \\ Erectile dysfunction; \\ Cardiovascular diseases; \\ Metabolic syndrome.}

\begin{abstract}
Summary
Background: A large body of evidences indicates that sexual dysfunction, and in particular erectile dysfunction (ED), may represent an early surrogate marker of different disease states such as diabetes mellitus, hypertension, metabolic syndrome (MetS) and depression. Furthermore, it has been suggested that ED could also be considered the first sign of a forthcoming coronary heart disease (CHD) and an efficient predictor of silent CHD in a diabetic population, independently of glycometabolic control and ED severity. Hypogonadism is frequently associated with MetS both in subjects with or without ED, insulin resistance being the putative pathogenetic link. In subjects with ED hypogonadism can exacerbate sexual dysfunction because of its typical symptoms, such as decreased sexual desire and mood disturbances. However, hypogonadism per se has been associated with an increased risk of cardiovascular and overall mortality.

Aim of the study: In this review, a comprehensive literature search was carried out, in order to discuss the relationship between insulin resistance, ED, MetS and hypogonadism, focusing on their possible involvement in the development of cardiovascular diseases.
\end{abstract}

(c) 2009 Elsevier Srl. All rights reserved.

\section{Introduzione}

La sindrome metabolica (SM) rappresenta una categoria diagnostica costituita da un gruppo di alterazioni metaboliche associate a uno stato di insulino-resistenza e a un

* Corrispondenza: UO di Endocrinologia e Malattie del Ricambio, Dipartimento Medico, Azienda USL di Bologna, Ospedale "MaggioreBellaria", l.go Nigrisoli 2 - 40133 Bologna.

E-mail: jocorona@libero.it (G. Corona). aumentato rischio di sviluppare patologie cardiovascolari (PCV) e metaboliche [1]. Negli ultimi anni, inoltre, è stata osservata un'associazione tra insulino-resistenza, SM e ipogonadismo maschile sia nei soggetti con disfunzione erettile (DE) [2-6] sia in quelli senza DE $[7,8]$. Recentemente la DE e l'ipogonadismo maschile sono stati entrambi considerati possibili segni precoci di PCV e SM $[9,10]$ e l'insulinoresistenza è stata proposta come il possibile elemento patogenetico comune sotteso a tali condizioni. 
Scopo del presente articolo è chiarire la relazione esistente tra insulino-resistenza, DE, obesità, SM e ipogonadismo maschile e il possibile coinvolgimento di tali condizioni nello sviluppo di PCV.

\section{Classificazione della sindrome metabolica}

Sebbene esistano diverse classificazioni diagnostiche di SM $[10,11]$, attualmente $\mathrm{i}$ criteri maggiormente impiegati sono quelli proposti dal National Cholesterol Education Program Third Adult Treatment Panel (NCEP-ATPIII) (tabella 1). Oltre alla classificazione NCEP-ATPIII ne esistono altre, per esempio quella della World Health Organitation del 1999 e dell'American College of Endocrinology (ACE) che si basano sulla presenza di insulino-resistenza o di intolleranza al glucosio, e quella sviluppata più recentemente dall'International Diabetes Federation (IDF), che assegna un ruolo centrale all'obesità viscerale.

\section{Definizione di ipogonadismo a insorgenza tardiva}

L'ipogonadismo a insorgenza tardiva ( $\mathrm{LOH})$, detto anche "sindrome da deficienza di testosterone" [12], è una condizione clinica che interessa frequentemente il maschio adulto-anziano, i cui meccanismi patogenetici e la cui storia clinica non sono ancora del tutto noti. Il sintomo più spesso associato a tale condizione è rappresentato da una riduzione del desiderio sessuale. Altri sintomi includono la DE, una diminuzione della massa e della forza muscolare, un incremento della massa grassa, una riduzione della densità ossea, nonché un calo della vitalità intesa in senso generale e la possibile presenza di depressione [12].

Recentemente, una consensus conference delle principali Società andrologiche internazionali ha rivisto i criteri diagnostici di LOH. In particolare, vi è unanime accordo sul fatto che la terapia sostitutiva con testosterone $(T)$ sia necessaria quando i livelli circolanti di T totale siano $<8 \mathrm{nmol} / \mathrm{L}(2,31$ $\mathrm{ng} / \mathrm{mL}$ ). Parimenti, vi è un'indicazione al non trattamento per valori di $\mathrm{T}>12 \mathrm{nmol} / \mathrm{L}(3,5 \mathrm{ng} / \mathrm{mL})$. Esiste un'area grigia compresa tra 8 e $12 \mathrm{nmol} / \mathrm{L}$ in cui è indicato il calcolo del T libero circolante secondo la formula di Vermeulen [12] (http://www. issam.ch/freetesto.htm).

Occorre infatti sottolineare che il T totale circola legato nel $50 \%$ circa a una proteina definita Sex Hormone Binding Globulin (SHBG). La solidità di tale legame impedisce che la quota di T trasportata dall'SHBG sia disponibile per l'attività biologica dell'ormone. L'SHBG è, a sua volta, influenzata da diversi fattori tra cui si sottolineano l'invecchiamento stesso e l'insulino-resistenza (tabella 2). Dal semplice dosaggio del $\mathrm{T}$ totale derivano possibili conseguenze negative sulla stima

Tabella 1 Definizione della sindrome metabolica secondo i criteri di: National Cholesterol Education Program-Third Adult Treatment Panel (NCEP-ATPIII), International Diabetes Federation (IDF), World Health Organization (WHO), American College of Endocrinology (ACE).

\begin{tabular}{|c|c|c|c|}
\hline NCEP-ATPIII & IDF & WHO & ACE \\
\hline $\begin{array}{l}3 \text { o più dei } \\
\text { seguenti criteri: }\end{array}$ & $\begin{array}{l}\text { Obesità centrale } \\
\text { (circonferenza } \\
\text { vita } \geq 94 \mathrm{~cm} \text { ) e } \\
2 \text { o più dei } \\
\text { seguenti criteri: }\end{array}$ & $\begin{array}{l}\text { Insulinemia a digiuno nel } \\
\text { quartile superiore; glucosio } \\
\text { a digiuno } \geq 100 \mathrm{mg} / \mathrm{dL} \\
(6,1 \mathrm{mmol} / \mathrm{L}) ; \text { glucosio } \\
\text { a } 2 \mathrm{ore} \geq 140 \mathrm{mg} / \mathrm{dL} \\
\text { (7,8 mmol/L) e } 2 \text { o } \\
\text { più dei seguenti criteri: }\end{array}$ & $\begin{array}{l}\text { Alto rischio di } \\
\text { resistenza insulinica: } \\
\text { glicemia plasmatica a } \\
2 \text { ore } \geq 140 \mathrm{mg} / \mathrm{dL} \\
(7,8 \mathrm{mmol} / \mathrm{L}) \text { e } \\
<200 \mathrm{mg} / \mathrm{dL}(11 \mathrm{mmol} / \mathrm{L}) \\
\text { e } 2 \text { o più dei seguenti criteri: }\end{array}$ \\
\hline $\begin{array}{l}\text { - Obesità centrale } \\
\text { (circonferenza } \\
\text { vita }>102 \mathrm{~cm} \text { ) } \\
\text { - Ipetrigliceridemia: } \\
\text { trigliceridi } \\
\geq 150 \mathrm{mg} / \mathrm{dL} \\
(1,7 \mathrm{mmol} / \mathrm{L}) \text { o } \\
\text { trattamento specifico } \\
\text { - Bassi livelli di } \\
\text { HDL: }<40 \mathrm{mg} / \mathrm{dL} \\
(1,03 \mathrm{mmol} / \mathrm{L}) \text { o } \\
\text { trattamento specifico } \\
\text { - Ipertensione arteriosa: } \\
\text { pressione arteriosa } \\
\geq 130 / 85 \mathrm{mmHg} 0 \\
\text { trattamento specifico } \\
\text { - Glicemia plasmatica } \\
\text { a digiuno: } \geq 110 \mathrm{mg} / \mathrm{dL} \\
\text { (6,1 mmol/L) o } \\
\text { diabete mellito }\end{array}$ & $\begin{array}{l}\text { - Ipertrigliceridemia: } \\
\text { trigliceridi } \\
\geq 150 \mathrm{mg} / \mathrm{dL} \\
(1,7 \mathrm{mmol} / \mathrm{L}) \text { o } \\
\text { trattamento specifico } \\
\text { - Bassi livelli di } \\
\text { colesterolo HDL: } \\
<40 \mathrm{mg} / \mathrm{dL} \\
(1,03 \mathrm{mmol} / \mathrm{L}) \text { o } \\
\text { trattamento specifico } \\
\text { - Ipertensione arteriosa: } \\
\text { pressione arteriosa } \\
\geq 130 / 85 \mathrm{mmHg} 0 \\
\text { trattamento specifico } \\
\text { - Glicemia plasmatica } \\
\text { a digiuno: } \geq 100 \mathrm{mg} / \mathrm{dL} \\
\text { (6,1 mmol/L) o } \\
\text { diabete mellito }\end{array}$ & $\begin{array}{l}\text { - Rapporto vita/fianchi } \\
>0,9 \text { o BMI } \geq 30 \mathrm{~kg} / \mathrm{m}^{2} \\
\text { - Ipertrigliceridemia: } \\
\text { trigliceridi } \geq 150 \mathrm{mg} / \mathrm{dL} \\
(1,7 \mathrm{mmol} / \mathrm{L}) \text { o } \\
\text { trattamento specifico } \\
\text { - Bassi livelli di colesterolo } \\
\mathrm{HDL}:<35 \mathrm{mg} / \mathrm{dL} \\
(0,9 \mathrm{mmol} / \mathrm{L}) \text { o } \\
\text { trattamento specifico } \\
\text { - Ipertensione arteriosa: } \\
\text { pressione arteriosa } \\
\geq 140 / 90 \text { mmHg o } \\
\text { trattamento specifico } \\
\text { - Microalbuminuria: } \\
\text { rapporto albumina urinaria/ } \\
\text { creatinina urinaria } \geq 3,39 \mathrm{mg} / \mathrm{mmol} \\
\text { (30 mg/g) }\end{array}$ & $\begin{array}{l}\text { - Ipertrigliceridemia: } \\
\text { trigliceridi } \geq 150 \mathrm{mg} / \mathrm{dL} \\
(1,7 \mathrm{mmol} / \mathrm{L}) \text { o } \\
\text { trattamento specifico } \\
\text { - Bassi livelli di colesterolo } \\
\text { HDL: }<40 \mathrm{mg} / \mathrm{dL} \\
(1,03 \mathrm{mmol} / \mathrm{L}) \text { o } \\
\text { trattamento specifico } \\
\text { - Ipertensione arteriosa: } \\
\text { pressione arteriosa } \\
\geq 130 / 85 \mathrm{mmHg} \text { o } \\
\text { trattamento specifico } \\
\text { - Glicemia plasmatica } \\
\text { a digiuno } \geq 100 \mathrm{mg} / \mathrm{dL} \\
(5,6 \mathrm{mmol} / \mathrm{L})\end{array}$ \\
\hline
\end{tabular}


Tabella 2 Principali fattori coinvolti nelle modificazioni dei livelli circolati di SHBG.

\section{Aumento di SHBG}

- Farmaci: antiepilettici, estrogeni, ormoni toroidei

- Ipertiroidismo

- Malattie epatiche

- Invecchiamento

Diminuzione di SHBG

- Obesità/insulino-resistenza

- Ipotiroidismo

- Acromegalia stati riportati nello European Prospective Investigation into Cancer in Norfolk Prospective Population Study [17]. Infine, più recentemente è stato riportato come l'ipogonadismo possa costituire un fattore di rischio per mortalità CV nella popolazione diabetica [18] e aumentare il rischio di ictus conclamato o attacchi ischemici transitori nella popolazione generale [19].

Nonostante i dati a disposizione siano limitati, è possibile sottolineare che il T esercita effetti positivi sulla reattività vascolare, sull'infiammazione, sulla produzione di citochine, sull'espressione di molecole di adesione e sui livelli sierici di lipidi e fattori della coagulazione, suggerendo un suo ruolo protettivo nello sviluppo dell'aterosclerosi e delle complicanze cliniche a essa correlate [10].

del grado di ipogonadismo (inteso come quota biologicamente attiva di T totale circolante). Pertanto, in presenza di valori di T totale compresi tra 8 e $12 \mathrm{nmol} / \mathrm{L}$ è consigliata la valutazione del testosterone libero calcolato secondo la formula di Vermeulen: per valori $<225 \mathrm{pmol} / \mathrm{L}$ è indicata una terapia sostitutiva. Viceversa, la valutazione del $T$ libero attraverso i normali metodi immunometrici è del tutto inattendibile e non dovrebbe essere eseguita [12].

\section{Sindrome metabolica e rischio cardiovascolare}

Il significato clinico della SM e in particolare la sua utilità nel predire il rischio cardiovascolare (CV) sono stati oggetto di numerose critiche, comprese quelle dell'American Diabetes Association e della European Association for the Study of Diabetes [11]. È stato sottolineato, infatti, come la SM di per sé debba essere considerata un cattivo predittore del rischio $\mathrm{CV}$ assoluto a breve termine, non contemplando, per definizione, alcuni importanti fattori di rischio come l'età, i livelli di colesterolo sierico, il sesso e l'abitudine al fumo [1]. Tuttavia la diagnosi di SM nei soggetti con rischio CV lievemoderato è da ritenersi utile, perché sottolinea la necessità di intervenire più efficacemente sullo stile di vita di questi individui, in modo da ritardare la progressione verso una categoria di rischio $\mathrm{CV}$ più elevata [1].

\section{Ipogonadismo e rischio cardiovascolare}

L'aspettativa di vita per il maschio negli Stati Uniti è di circa 6 anni inferiore rispetto a quella della femmina [13]. Sebbene le cause di tali differenze non siano state ancora del tutto comprese, negli ultimi dieci anni è stato ipotizzato che il T possa svolgere un ruolo causale nel determinare un'aspettativa di vita inferiore nei maschi.

Studi pubblicati negli ultimi quattro anni hanno notevolmente modificato tale visione. Dati derivanti dal Massachusetts Male Aging Study (MMAS) [14] e dal Caerphilly Study [15] non hanno dimostrato alcuna associazione fra livelli di Te mortalità globale. I dati longitudinali dello studio Rancho Bernardo suggeriscono che i soggetti con livelli di T totale nel quartile più basso $(<2,4 \mathrm{ng} / \mathrm{mL} ; 8,1 \mathrm{nmol} / \mathrm{L})$ siano caratterizzati da un aumento di mortalità globale e CV del $40 \%$ e del $38 \%$ rispettivamente, quando confrontati con il resto della popolazione [16]. Risultati simili sono

\section{Disfunzione erettile e rischio cardiovascolare}

Diverse evidenze cliniche suggeriscono che la DE possa rappresentare un possibile segno precoce di patologia coronarica (PC). In particolare, è stato dimostrato come in soggetti con PC documentata mediante angiografia, l'insorgenza della DE preceda di circa 3 anni la comparsa di un evento cardiovascolare nel 70\% circa dei casi [9]. Nel Prostate Cancer Prevention Trial è stato documentato, seguendo alcuni pazienti per un periodo di 9 anni dall'esordio della DE, come la comparsa di DE fosse associata a un incremento del $25 \%$ del rischio di sviluppare un successivo evento $\mathrm{CV}$, anche dopo correzione per fattori confondenti [20]. Inoltre, è stato suggerito che la DE possa essere considerata il miglior segno predittivo per una PC silente nella popolazione diabetica, indipendentemente dal controllo glicometabolico e dalla severità della DE [21]. Recentemente abbiamo dimostrato come i valori di velocità di picco sistolico misurati campionando le arterie cavernose del pene in condizioni di flaccidità possano rappresentare un buon indice per predire la presenza di una PC silente nella popolazione diabetica, mostrando un'accuratezza dell'80\% [21].

La disfunzione endoteliale costituisce l'anello di connessione comune tra DE e PC. Secondo l'ipotesi del calibro arterioso [9], le arterie cavernose del pene, di piccolo calibro, in condizioni patologiche raggiungono livelli di stenosi critici, associati a ipoafflusso ematico relativo, precocemente rispetto a vasi di calibro maggiore, come quelli del distretto coronarico, i vasi epiaortici (carotidi) o quelli degli arti inferiori (ileo-femorali). Inoltre anche l'ipogonadismo, che spesso è un cofattore nella patogenesi della DE [10], può contribuire al meccanismo patologico.

\section{Meccanismi patogenetici}

\section{Ipogonadismo e sindrome metabolica}

Diverse evidenze cliniche hanno documentato una stretta associazione tra SM e ipogonadismo, anche nei soggetti che riferiscono un disturbo della sessualità $[2-8,10]$. D'altro canto la SM si associa a un'aumentata prevalenza di DE, caratterizzata essenzialmente da una maggiore compromissione del flusso sanguigno penieno $[2-8,10]$. Tale dato non dovrebbe sorprendere, perché, sebbene l'insulina in 
concentrazioni fisiologiche nei soggetti sani eserciti un'azione acuta vasodilatatrice, che si associa a un'aumentata distensibilità vascolare, questo benefico effetto è perso in condizioni di insulino-resistenza, come nella SM [10,21]. Dati ottenuti nello studio longitudinale MMAS hanno dimostrato come la DE possa perfino rappresentare un elemento predittivo dello sviluppo di SM nei soggetti normopeso [22].

Tra i fattori che costituiscono la SM, l'elevata circonferenza della vita e l'ipertrigliceridemia (parametri maggiormente associati a una condizione di insulino-resistenza) rappresentano i maggiori determinanti dell'ipogonadismo [2-5,10,21]. Viceversa, la relazione tra ipogonadismo e ipertensione arteriosa è molto più discussa [10]. Recentemente abbiamo dimostrato una forte relazione inversa tra T non legato alla SHBG e pressione differenziale (PD) [23]. La PD è la differenza aritmetica tra pressione arteriosa sistolica e pressione arteriosa diastolica e riflette la rigidità delle arterie, altro indice di insulino-resistenza [23].

\section{Ipogonadismo e controllo glicemico}

Sia i dati ottenuti da modelli animali sia quelli ricavati da studi epidemiologici suggeriscono che il $\mathrm{T}$ giochi un ruolo cruciale nella regolazione della sensibilità all'insulina. Studi prospettici hanno chiaramente dimostrato che il riscontro di bassi livelli di T può predire lo sviluppo sia di diabete mellito (DM) di tipo 2 sia di SM [10]. Yialamas et al. [24] hanno inoltre confermato che la sospensione improvvisa della terapia sostitutiva con T si associa a una riduzione della sensibilità all'insulina, senza osservare variazioni nella composizione corporea.

In accordo con tali osservazioni, studi condotti su soggetti trattati per carcinoma prostatico mediante soppressione androgenica a lungo termine ( $\geq 12$ mesi) hanno documentato una maggiore prevalenza di DM e SM e una più alta mortalità per eventi CV [25]. D'altronde il trattamento con $T$ di soggetti diabetici ipogonadici migliora l'insulino-resistenza, sebbene studi controllati abbiano valutato solo una serie limitata di soggetti [21].

\section{Ipogonadismo e obesità}

L'obesità, e in particolare l'obesità centrale, rappresenta l'elemento chiave della SM.

L'obesità di per sé, indipendentemente dai disordini metabolici associati, è notoriamente in relazione con una riduzione dei livelli di SHBG $[10,26]$, con inevitabili conseguenze sul $\mathrm{T}$ totale circolante (possibilità di sovrastimare l'ipogonadismo, si veda sopra). Tuttavia è stato dimostrato come i livelli sia di T legato sia di T non legato alla SHBG diminuiscano nei maschi obesi proporzionalmente al loro grado di obesità, anche dopo correzione per le condizioni a essa associate [5,27]. Inoltre, soggetti con obesità morbigena mostrano una riduzione dell'ampiezza della pulsatilità dell'ormone luteinizzante quando confrontati con soggetti di controllo normopeso [28,29]. Tali osservazioni supportano il concetto di un ipoandrogenismo obesità-correlato.

L'ipogonadismo, pertanto, può essere considerato una delle numerose complicanze del sovrappeso e dell'obesità. D'altra parte, bassi livelli di $\mathrm{T}$ possono svolgere un ruolo nell'accumulo di grasso in eccesso, stabilendo così un circolo vizioso. I dati derivanti dal MMAS [30] hanno documentato, in effetti, un maggiore declino del T totale e libero in soggetti costantemente obesi nel corso di un follow-up di 8-9 anni. Inoltre, modificazioni dello stile di vita finalizzate a ottenere un calo ponderale si accompagnano a un miglioramento del profilo CV e a un aumento dei livelli di T circolante [10,21]. Quale molecola giochi il ruolo principe in questo circolo vizioso non è ancora noto, ma i possibili candidati sono rappresentati da estrogeni, insulina, leptina, TNF-alfa o altre adipochine $[10,21,26]$.

\section{Conclusioni}

L'ipogonadismo, la DE, l'obesità, l'insulino-resistenza e la SM sono condizioni cliniche frequentemente associate nello stesso individuo. Questo gruppo di affezioni è caratterizzato da un aumentato rischio di sviluppare patologie CV e metaboliche, con un impatto rilevante non solo sulla qualità ma anche sull'aspettativa di vita dei pazienti.

Nei pazienti con DE tale sintomo offre l'occasione per approfondire, trattare o prevenire patologie sottostanti potenzialmente pericolose. Sebbene risultati preliminari possano suggerire una riduzione del rischio CV dopo terapia sostitutiva con testosterone, sono necessari ulteriori studi per poter confermare i risultati incoraggianti attualmente disponibili.

\section{Conflitto d'interessi}

Gli autori dichiarano di essere esenti da conflitto di interessi.

\section{Bibliografia}

[1] Grundy SM. Metabolic syndrome: a multiplex cardiovascular risk factor. J Clin Endocrinol Metab 2007;92(2):399-404.

[2] Corona G, Mannucci E, Schulman C, Petrone L, Mansani R, Cilotti A, et al. Psychobiologic correlates of the metabolic syndrome and associated sexual dysfunction. Eur Urol 2006;50(3):595-604.

[3] Corona G, Mannucci E, Petrone L, Balercia G, Paggi F, Fisher AD, et al. NCEP-ATPIII-defined metabolic syndrome, type 2 diabetes mellitus, and prevalence of hypogonadism in male patients with sexual dysfunction. J Sex Med 2007;4(4 Pt 1):1038-45.

[4] Corona G, Mannucci E, Petrone L, Schulman C, Balercia G, Fisher $A D$, et al. A comparison of NCEP-ATPIII and IDF metabolic syndrome definitions with relation to metabolic syndrome-associated sexual dysfunction. J Sex Med 2007;4(3):789-96.

[5] Corona G, Mannucci E, Fisher AD, Lotti F, Petrone L, Balercia G, et al. Low levels of androgens in men with erectile dysfunction and obesity. J Sex Med 2008;5(10):2454-63.

[6] Guay A, Jacobson J. The relationship between testosterone levels, the metabolic syndrome (by two criteria), and insulin resistance in a population of men with organic erectile dysfunction. J Sex Med 2007;4(4 Pt 1):1046-55.

[7] Traish AM, Saad F, Guay A. The dark side of testosterone deficiency: II. Type 2 diabetes and insulin resistance. J Androl 2009;30(1):23-32.

[8] Maggi M, Schulman C, Quinton R, Langham S, Uhl-Hochgraeber $\mathrm{K}$. The burden of testosterone deficiency syndrome in adult men: economic and quality-of-life impact. J Sex Med 2007;4(4 Pt 1):1056-69.

[9] Montorsi P, Ravagnani PM, Galli S, Salonia A, Briganti A, Werba $J P$, et al. Association between erectile dysfunction and coronary 
artery disease: Matching the right target with the right test in the right patient. Eur Urol 2006;50(4):721-31.

[10] Corona G, Mannucci E, Forti G, Maggi M. Hypogonadism, ED, metabolic syndrome and obesity: a pathological link supporting cardiovascular diseases. Int J Androl 2009. [Epub ahead of print.]

[11] Kahn R, Buse J, Ferrannini E, Stern M, American Diabetes Association, European Association for the Study of Diabetes. The metabolic syndrome: time for a critical appraisal: joint statement from the American Diabetes Association and the European Association for the Study of Diabetes. Diabetes Care 2005;28(9):2289-304.

[12] Wang C, Nieschlag E, Swerdloff R, Behre HM, Hellstrom WJ, Gooren LJ, et al. Investigation, treatment, and monitoring of late-onset hypogonadism in males: ISA, ISSAM, EAU, EAA, and ASA Recommendations. Eur Urol 2008. [Epub ahead of print.]

[13] Courtenay WH, Keeling RP. Men, gender, and health: toward an interdisciplinary approach. J Am Coll Health 2000;48(6):243-6.

[14] Araujo AB, Kupelian V, Page ST, Handelsman DJ, Bremner WJ, McKinlay JB. Sex steroids and all-cause and cause-specific mortality in men. Arch Intern Med 2007;167(12):1252-60.

[15] Smith GD, Ben-Shlomo Y, Beswick A, Yarnell J, Lightman S, Elwood P. Cortisol, testosterone, and coronary heart disease: prospective evidence from the Caerphilly Study. Circulation 2005;112(3):332-40.

[16] Laughlin GA, Barrett-Connor E, Bergstrom J. Low serum testosterone and mortality in older men. J Clin Endocrinol Metab 2008;93(1):68-75.

[17] Khaw KT, Dowsett M, Folkerd E, Bingham S, Wareham N, Luben R, et al. Endogenous testosterone and mortality due to all causes, cardiovascular disease, and cancer in men: European Prospective Investigation into Cancer in Norfolk (EPIC-Norfolk) Prospective Population Study. Circulation 2007;116(23):2694-701.

[18] Ponikowska B, Jankowska EA, Maj J, Wegrzynowska-Teodorczyk $\mathrm{K}$, Biel B, Reczuch K, et al. Gonadal and adrenal androgen deficiencies as independent predictors of increased cardiovascular mortality in men with type II diabetes mellitus and stable coronary artery disease. Int J Cardiol 2009. [Epub ahead of print.]

[19] Yeap BB, Hyde Z, Almeida OP, Norman PE, Chubb SA, Jamrozik K, et al. Lower testosterone levels predict incident stroke and transient ischemic attack in older men. J Clin Endocrinol Metab 2009;94(7):2353-9.
[20] Thompson IM, Tangen CM, Goodman PJ, Probstfield JL, Moinpour CM, Coltman CA. Erectile dysfunction and subsequent cardiovascular disease. JAMA 2005;294(23):2996-3002.

[21] Corona G, Mannucci E, Forti G, Maggi M. Following the common association between testosterone deficiency and diabetes mellitus, can testosterone be regarded as a new therapy for diabetes? Int J Androl 2009 [Epub ahead of print.]

[22] Kupelian V, Shabsigh R, Araujo AB, O’Donnell AB, McKinlay JB. Erectile dysfunction as a predictor of the metabolic syndrome in aging men: results from the Massachusetts Male Aging Study. J Urol 2006;176(1):222-6.

[23] Corona G, Mannucci E, Lotti F, Fisher AD, Bandini E, Balercia G, et al. Pulse pressure, an index of arterial stiffness, is associated with androgen deficiency and impaired penile blood flow in men with ED. J Sex Med 2009;6(1):285-93.

[24] Yialamas MA, Dwyer AA, Hanley E, Lee H, Pitteloud N, Hayes FJ. Acute sex steroid withdrawal reduces insulin sensitivity in healthy men with idiopathic hypogonadotropic hypogonadism. $\mathrm{J}$ Clin Endocrinol Metab 2007;92(11):4254-9.

[25] Basaria S. Androgen deprivation therapy, insulin resistance, and cardiovascular mortality: an inconvenient truth. J Androl 2008;29(5):534-9.

[26] Pitteloud N, Hardin M, Dwyer AA, Valassi E, Yialamas M, Elahi D, et al. Increasing insulin resistance is associated with a decrease in Leydig cell testosterone secretion in men. J Clin Endocrinol Metab 2005;90(5):2636-41.

[27] Zumoff B, Strain GW, Miller LK, Rosner W, Senie R, Seres DS, et al. Plasma free and non-sex-hormone-binding-globulinbound testosterone are decreased in obese men in proportion to their degree of obesity. J Clin Endocrinol Metab 1990;71(4): 929-31.

[28] Giagulli VA, Kaufman JM, Vermeulen A. Pathogenesis of the decreased androgen levels in obese men. J Clin Endocrinol Metab 1994;79(4):997-1000.

[29] Vermeulen A, Kaufman JM, Deslypere JP, Thomas G. Attenuated luteinizing hormone (LH) pulse amplitude but normal LH pulse frequency, and its relation to plasma androgens in hypogonadism of obese men. J Clin Endocrinol Metab 1993;76(5): 1140-6.

[30] Derby CA, Zilber S, Brambilla D, Morales KH, McKinlay JB. Body mass index, waist circumference and waist to hip ratio and change in sex steroid hormones: the Massachusetts Male Ageing Study. Clin Endocrinol (Oxf) 2006;65(1):125-31. 